\title{
Fenologia de floração e sistema reprodutivo de três espécies de Ruellia (Acanthaceae) em fragmento florestal de Viçosa, Sudeste brasileiro ${ }^{1}$
}

\author{
NATÁLIA A. SOUZA LIMA² e MILENE FARIA VIEIRA ${ }^{3,4}$
}

(recebido: 17 de novembro de 2005; aceito: 16 de novembro de 2006)

\begin{abstract}
Flowering phenology and breeding system of three species of Ruellia (Acanthaceae) in a forest fragment in Viçosa, southeastern Brazil). This work is a continuation of studies on the reproductive biology of angiosperm species in the municipality of Viçosa, "Zona da Mata", Minas Gerais State, Brazil. The aim of this work was to analyze the flowering phenology and the breeding system of three co-occurring species of Ruellia: R. brevifolia (Pohl) C. Ezcurra and R. menthoides (Nees) Hiern, both cleistogamous, and R. subsessilis (Nees) Lindau. Seed germination was tested and the occurrence of vegetative propagation verified. Ruellia subsessilis flowered throughout the year, with a peak in October. Ruellia brevifolia produced flowers mainly from February to July, with a flowering peak in March. Ruellia menthoides produced flowers from August to December with a flowering peak in September. All species are self-compatible; Ruellia brevifolia and R. menthoides are facultative autogamous species and $R$. subsessilis is a facultative xenogamous species. High percentages of normal seedlings (from $67 \%$ up to $83 \%$ ) were obtained from seeds produced through selfing. Besides sexual reproduction, the species showed vegetative propagation, i.e., branch fragments segregated from the motherplant, which develop adventitious roots. The species, therefore, showed sexual and asexual reproductive strategies. The former are distinct flowering peak, autogamous or allogamous flowers as well as ornitophilous or mellittophilous ones, and cleistogamous flowers. However, the result of sexual strategies was common to all of them, i.e., production of fruits with viable seeds during the entire year. The balance among the various reproductive mechanisms seems to confer to these species a high degree of adaptation in altered environments as shown in areas of their occurrence at the forest fragment studied.
\end{abstract}

Key words - flowering phenology, Ruellia brevifolia, Ruellia menthoides, Ruellia subsessilis, self-compatibility

RESUMO - (Fenologia de floração e sistema reprodutivo de três espécies de Ruellia (Acanthaceae) em fragmento florestal de Viçosa, Sudeste brasileiro). Este trabalho é uma continuação de estudos sobre a biologia reprodutiva de espécies de angiospermas do Município de Viçosa, Zona da Mata de Minas Gerais, Brasil. Objetivou-se analisar a fenologia de floração e o sistema reprodutivo das três espécies co-ocorrentes de Ruellia observadas nesse município: R. brevifolia (Pohl) C. Ezcurra e R. menthoides (Nees) Hiern, ambas cleistógamas, e $R$. subsessilis (Nees) Lindau. Testou-se a germinação de suas sementes e verificou-se a ocorrência de propagação vegetativa. A floração de Ruellia subsessilis ocorreu durante todo o ano, sendo o pico de floração em outubro. Ruellia brevifolia produziu flores, principalmente, de fevereiro a julho, com o pico em março. Ruellia menthoides produziu flores de agosto a dezembro, com o pico em setembro. As espécies são autocompatíveis; Ruellia brevifolia e $R$. menthoides autógamas facultativas e $R$. subsessilis xenógama facultativa. Obtiveram-se altas porcentagens de plântulas normais (de 67\% a 83\%), de sementes originadas de autopolinizações. Além da reprodução sexuada, as espécies apresentaram propagação vegetativa, ou seja, pedaços de ramos separam-se das plantas-mãe e desenvolvem raízes adventícias. As espécies estudadas, portanto, apresentaram estratégias reprodutivas sexuadas e assexuadas. As sexuadas consistem de períodos distintos de picos de floração, flores casmógamas autógamas ou alógamas, ornitófilas ou melitófilas e flores cleistógamas. Entretanto, o resultado das estratégias sexuadas foi comum a todas elas, ou seja, produção de frutos com sementes viáveis durante todo o ano. $\mathrm{O}$ balanço entre os mecanismos variados de reprodução parece conferir a essas espécies elevado grau de adaptação a ambientes alterados, como é o caso das áreas de sua ocorrência, no fragmento florestal do presente estudo.

Palavras-chave - autocompatibilidade, fenologia de floração, Ruellia brevifolia, Ruellia menthoides, Ruellia subsessilis

\section{Introdução}

O gênero Ruellia, um dos maiores da família Acanthaceae, compreende aproximadamente 250

1. Parte da dissertação de mestrado da primeira autora, Programa de Pós-Graduação em Botânica, Universidade Federal de Viçosa, Viçosa, MG, Brasil.

2. Escritório Regional do Ibama de Parintins, Rua Paes de Andrade 747, Centro, 69151-200 Parintins, AM, Brasil.

3. Universidade Federal de Viçosa, Departamento de Biologia Vegetal, 36570-000 Viçosa, MG, Brasil.

4. Autor para correspondência: mfvieira@ufv.br espécies, amplamente distribuídas nas regiões tropicais e subtropicais, especialmente do Novo Mundo, seu principal centro de diversidade (Ezcurra 1993). No Município de Viçosa, Zona da Mata do Estado de Minas Gerais, local do presente estudo, foram reconhecidas três espécies de Ruellia: R. brevifolia (Pohl) C. Ezcurra, $R$. menthoides (Nees) Hiern e R. subsessilis (Nees) Lindau (Braz et al. 2002). A primeira é amplamente distribuída na América do Sul (Ezcurra 1993) e as outras duas têm distribuição restrita ao território nacional (Nees 1847, Braz et al. 2002). Ruellia 
subsessilis foi originalmente citada para o Estado de Minas Gerais (Nees 1847).

A vegetação original de Viçosa era constituída por florestas contínuas, inseridas nos domínios da Floresta Atlântica (Rizzini 1992). Atualmente, é composta de pastagens e poucos fragmentos florestais, em diversos estádios sucessionais e, por isso, tem sido considerada prioritária para investigação científica. Apesar do elevado grau de devastação da flora local, estudos realizados sobre a biologia reprodutiva de espécies remanescentes têm contribuído para o conhecimento da flora brasileira, com informações relevantes sobre morfologia floral (Vieira \& Shepherd 2002), polinizadores (Vieira \& Carvalho-Okano 1996, Vieira \& Lima 1997) e sistemas reprodutivos (Vieira \& Lima 1997, Vieira \& Grabalos 2003).

As três espécies de Ruellia, em Viçosa, habitam o sub-bosque e $R$. brevifolia e $R$. menthoides são cleistógamas, mecanismo reprodutivo verificado em, pelo menos, 15 espécies desse gênero (Lima et al. 2005). Além das espécies de Ruellia, a maioria das espécies herbáceas e lenhosas de sub-bosque tem apresentado autocompatibilidade (MacDade 1985, Kress \& Beach 1994).

Diversas Acanthaceae são ornitófilas, incluindo espécies de Ruellia (Machado \& Sazima 1995, Piovano et al. 1995, Braz et al. 2000, Sigrist \& Sazima 2002, Abreu \& Vieira 2004). Em Viçosa, essa família contribui, dentre as espécies ornitófilas conhecidas, com o maior número de espécies (5) visitadas por beija-flores, sendo $R$. brevifolia e Geissomeria schottiana Nees consideradas as principais fontes de néctar para essas aves durante a estação seca (Abreu \& Vieira 2004). Estudos sobre a fenologia de floração de espécies congêneres co-ocorrentes ou de espécies de uma mesma família, visitadas por animais antófilos em comum, auxiliam na compreensão de interações entre espécies, na comunidade. Além disso, esses estudos podem esclarecer fatores que mantêm o isolamento desses táxons (Stace 1989).

Objetivou-se, no presente trabalho, analisar a fenologia de floração e o sistema reprodutivo das três espécies de Ruellia ocorrentes em Viçosa. Considerando que $R$. brevifolia e várias outras espécies brasileiras desse gênero têm sido utilizadas como ornamentais (Lorenzi \& Souza 2001), esse estudo também visou fornecer dados sobre a propagação (sexuada ou assexuada) dessas plantas, em especial de $R$. subsessilis que também possui potencial ornamental, para produção de mudas. Esse estudo amplia o conhecimento sobre a flora remanescente de Viçosa e, pelo seu caráter de pesquisa básica, subsidia futuras estratégias para a conservação da vegetação local, que é fragmentária.

\section{Material e métodos}

Local de estudo - O presente trabalho foi realizado na Estação de Pesquisa, Treinamento e Educação Ambiental Mata do Paraíso (EPTEAMP), maior fragmento do Município de Viçosa (204'07' S e 42 51'31' W), com aproximadamente 195 ha. Sua vegetação pertence aos domínios da Floresta Atlântica, sendo classificada por Veloso et al. (1991) como Floresta Estacional Semidecidual Submontana. No século XIX, a área dessa reserva foi desmatada e utilizada para cultivos e pastagens (Leal Filho 1992). Atualmente, a mata existente é de regeneração secundária (Castro 1980).

O clima de Viçosa caracteriza-se por apresentar verões quentes e úmidos e invernos frios e secos (Leal Filho 1992). A média pluviométrica, nos anos de 1991 a 1999, foi de $1248,7 \mathrm{~mm}^{2} \mathrm{ano}^{-1}$, a temperatura média de $20^{\circ} \mathrm{C}$ e a umidade relativa do ar de aproximadamente $81 \%$ (Soares Júnior 2000).

Para estudos complementares, indivíduos das três espécies, oriundos da EPTEAMP, foram cultivados no Horto Botânico da Universidade Federal de Viçosa (UFV).

Espécies estudadas - Ruellia brevifolia (Pohl) C. Ezcurra é ornitófila (Piovano et al. 1995, Braz et al. 2000, Sigrist \& Sazima 2002, Abreu \& Vieira 2004), herbácea, com cerca de 1,0 m de altura, e suas inflorescências são axilares e sustentam flores vermelhas casmógamas (CA) e flores brancas cleistógamas (CL) (Piovano et al. 1995, Sigrist \& Sazima 2002, Lima et al. 2005). Na EPTEAMP, foi encontrada principalmente em locais abertos a parcialmente sombreados e os indivíduos, geralmente, encontravam-se agrupados.

Ruellia menthoides (Nees) Hiern é herbácea, com 0,5 a $0,8 \mathrm{~m}$ de altura, e suas inflorescências são axilares e sustentam flores brancas CA e CL (Lima et al. 2005); as CA são raramente visitadas pelos polinizadores, ou seja, pequenas abelhas (Lima 2001). Na EPTEAMP, foi observada em locais abertos a parcialmente sombreados, especialmente ao longo da trilha principal.

Ruellia subsessilis (Nees) Lindau é subarbustiva, com 0,7 a 1,5 m de altura, e suas inflorescências são axilares e sustentam flores rosas CA (Lima et al. 2005), visitadas por beija-flores (Braz et al. 2000, Abreu \& Vieira 2004). No local de estudo, foi encontrada principalmente em áreas sombreadas e os indivíduos, geralmente, encontravam-se agrupados.

Material testemunho das espécies estudadas foi depositado no Herbário VIC (números 23.745, 24.910 e 25.213), do Departamento de Biologia Vegetal da UFV.

Fenologia de floração - Foi acompanhada de fevereiro a dezembro de 2000. Todas as flores CA, em antese, de $R$. brevifolia e $R$. menthoides foram contadas em 198 e 165 indivíduos, respectivamente, localizados às margens da trilha principal da reserva, numa extensão de $600 \mathrm{~m}$, para cada espécie. As flores de R. subsessilis foram contadas em 328 
indivíduos localizados numa área com cerca de $350 \mathrm{~m}^{2}$. As contagens foram feitas semanalmente entre $7 \mathrm{~h} 00$ e $11 \mathrm{~h} 30$, período no qual as flores abrem-se ou já se encontram abertas (Lima 2001, Lima et al. 2005), exceto em abril, maio e julho em $R$. brevifolia e $R$. menthoides (três contagens em maio e julho e duas em abril) e em fevereiro e julho em $R$. subsessilis (três contagens em cada mês). O período de produção de flores CL, em $R$. brevifolia e $R$. menthoides, foi registrado por Lima et al. (2005). A ausência ou presença de frutos (imaturos ou maduros) foi acompanhada durante o período de estudo.

Sistema reprodutivo - Com base na metodologia de Dafni (1992), foram realizados os seguintes tratamentos, em flores CA das espécies estudadas: polinização aberta, polinização aberta em flores emasculadas, polinização cruzada, autopolinização espontânea, autopolinização manual e agamospermia. Devido à dificuldade em manusear as flores de $R$. menthoides, o número utilizado em alguns tratamentos foi baixo. Foi verificada a frutificação de flores CL em $R$. brevifolia e $R$. menthoides.

Foi testada a viabilidade de sementes originadas da autopolinização manual e espontânea em flores CA de $R$. brevifolia e $R$. subsessilis e autopolinização de flores CL de $R$. brevifolia e $R$. menthoides. Sementes foram colocadas em recipientes plásticos (gerbox) contendo papel de filtro umedecido e mantido em germinador a $25^{\circ} \mathrm{C}$. Foram consideradas viáveis as sementes que originaram plântulas com radícula, hipocótilo e cotilédones.

Foi verificada a ocorrência de propagação vegetativa nas espécies estudadas.

\section{Resultados}

Fenologia de floração - A floração de Ruellia brevifolia (Pohl) C. Ezcurra ocorreu durante quase todo o ano, com breve interrupção entre julho e setembro, e apresentou tendência sazonal para maior produção de flores em março (figura 1). A floração de Ruellia subsessilis (Nees) Lindau ocorreu durante todo o ano e apresentou tendência para maior produção de flores em outubro (figura 1). Ruellia menthoides (Nees) Hiern floresceu de agosto a dezembro, com um pico de floração em setembro (figura 1). Frutos foram observados em todas as espécies e durante todo o período de floração. Sistema reprodutivo - Os resultados corroboraram que as espécies de Ruellia são autocompatíveis (tabela 1). As frutificações obtidas nas polinizações abertas, se comparadas às obtidas nas polinizações abertas em flores emasculadas, indicam que os polinizadores têm sido pouco efetivos, especialmente em $R$. menthoides. Os maiores sucessos de frutificação, observados nas polinizações abertas, podem ser atribuídos à ocorrência de autopolinizações espontâneas, além das polinizações realizadas pelos polinizadores, e revela a importância

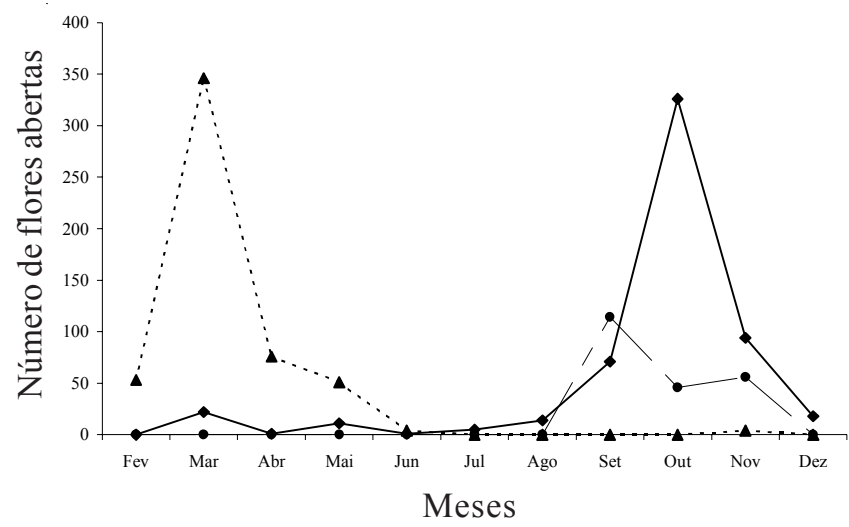

Figura 1. Floração de espécies de Ruellia, em Viçosa, MG, sudeste brasileiro, em 2000.

Figure 1. Flowering of the Ruellia species, in Viçosa, MG, southeastern Brazil, in 2000.

(- - $\mathbf{-}$ - - = Ruellia brevifolia (Pohl) C. Ezcurra;- - - = Ruellia subsessilis (Nees) Lindau;---•--- = Ruellia menthoides (Nees) Hiern).

da autogamia para as espécies estudadas. Apesar disso, o baixo número de flores abertas por indivíduo por dia (tabela 2) deve favorecer a xenogamia.

As maiores frutificações obtidas, em todas as espécies, nas autopolinizações manuais, se comparadas com as obtidas nas autopolinizações espontâneas (tabela 1), demonstram a existência de mecanismos que dificultam a autopolinização espontânea, especialmente em $R$. menthoides.

As frutificações das flores CL de $R$. brevifolia e $R$. menthoides foram altas (tabela 1). A agamospermia não foi observada nas espécies estudadas, exceto em $R$. brevifolia; entretanto, o único fruto registrado nessa espécie, provavelmente, resultou de uma polinização manual acidental.

Foram obtidas altas porcentagens de germinação com plântulas normais (tabela 3). A maior parte das anormalidades verificadas em plântulas das três espécies foi decorrente, principalmente, da contaminação primária por fungos e, ou bactérias.

Ruellia brevifolia, $R$. menthoides e $R$. subsessilis apresentaram propagação vegetativa, ou seja, ramos, da planta-mãe, quebram-se e separam-se dela; nesses ramos há desenvolvimento de raízes adventícias, possibilitando o estabelecimento dos clones.

\section{Discussão}

Fenologia de floração - O longo período de floração de Ruellia brevifolia (Pohl) C. Ezcurra e Ruellia 
Tabela 1. Resultados das polinizações em Ruellia brevifolia (Pohl) C. Ezcurra, R. menthoides (Nees) Hiern e R. subsessilis (Nees) Lindau, em Viçosa, MG, sudeste brasileiro. (PA = polinização aberta; $\mathrm{PAE}=$ polinização aberta em flores emasculadas; $\mathrm{PC}=$ polinização cruzada; $\mathrm{AUTe}=$ autopolinização espontânea; $\mathrm{AUTm}$ = autopolinização manual; $\mathrm{AUTcl}=$ autopolinização em flores cleistógamas; $\mathrm{AG}=$ agamospermia).

Table 1. Results of pollination in Ruellia brevifolia (Pohl) C. Ezcurra, R. menthoides (Nees) Hiern and R. subsessilis (Nees) Lindau, in Viçosa, MG, southeastern Brazil. $(\mathrm{PA}=$ open pollination; $\mathrm{PAE}=$ open pollination in emasculated flowers; $\mathrm{PC}=$ cross pollination; $\mathrm{AUTe}=$ spontaneous self-pollination; $\mathrm{AUTm}=$ manual self-pollination; $\mathrm{AUTcl}$ = self-pollination in cleistogamous flowers; $\mathrm{AG}=$ agamospermy).

\begin{tabular}{|c|c|c|c|c|c|c|c|c|c|}
\hline \multirow[t]{2}{*}{ Testes } & \multicolumn{3}{|c|}{ R. brevifolia } & \multicolumn{3}{|c|}{ R. menthoides } & \multicolumn{3}{|c|}{ R. subsessilis } \\
\hline & $\begin{array}{c}\text { Indivíduo } \\
\mathrm{n}^{\mathrm{o}}\end{array}$ & $\begin{array}{c}\text { Flor } \\
\mathrm{n}^{\mathrm{o}}\end{array}$ & $\begin{array}{l}\text { Fruto } \\
\mathrm{n}^{\circ}(\%)\end{array}$ & $\begin{array}{c}\text { Indivíduo } \\
\mathrm{n}^{\circ}\end{array}$ & $\begin{array}{c}\text { Flor } \\
\mathrm{n}^{\mathrm{o}}\end{array}$ & $\begin{array}{l}\text { Fruto } \\
\mathrm{n}^{\circ}(\%)\end{array}$ & $\begin{array}{c}\text { Indivíduo } \\
\mathrm{n}^{\mathrm{o}}\end{array}$ & $\begin{array}{c}\text { Flor } \\
\mathrm{n}^{\mathrm{o}}\end{array}$ & $\begin{array}{l}\text { Fruto } \\
\mathrm{n}^{\mathrm{o}}(\%)\end{array}$ \\
\hline PA & 18 & 34 & $26(76,47)$ & 16 & 21 & $14(66,67)$ & 21 & 29 & $18(62,07)$ \\
\hline PAE & 25 & 38 & $17(44,74)$ & 07 & 07 & $01(14,28)$ & 17 & 26 & $11(42,31)$ \\
\hline $\mathrm{PC}$ & 09 & 10 & $10(100,00)$ & 05 & 05 & $05(100,00)$ & 13 & 14 & $12(85,71)$ \\
\hline AUTe & 14 & 19 & $08(42,10)$ & 09 & 09 & $00(00,00)$ & 12 & 20 & $09(45,00)$ \\
\hline AUTm & 10 & 12 & $08(66,67)$ & 07 & 10 & $05(50,00)$ & 07 & 16 & $14(87,50)$ \\
\hline AUTcl & 11 & 20 & $19(95,00)$ & 09 & 26 & $25(96,15)$ & - & - & - \\
\hline AG & 13 & 15 & $01(06,67)$ & 09 & 11 & $00(00,00)$ & 14 & 21 & $00(00,00)$ \\
\hline
\end{tabular}

subsessilis (Nees) Lindau, associado à polinização por beija-flores (Braz et al. 2000, Sigrist \& Sazima 2002, Abreu \& Vieira 2004), permite enquadrá-las no padrão de floração "contínuo", da classificação de Newstrom et al. (1994). Por serem visitadas pelos mesmos beijaflores, conforme Braz et al. (2000) e Abreu \& Vieira (2004), o desencontro dos seus picos de floração, como verificado no presente estudo, deve reduzir a competição por polinizadores e a possibilidade de cruzamentos interespecíficos (Stiles 1975, Gentry 1974, 1990). Entre julho e setembro, meses nos quais $R$. brevifolia não produziu flores $\mathrm{CA}$, foi observada abundante produção de flores CL (Lima et al. 2005), o que resulta em uma frutificação contínua, assim como foi a de R. subsessilis.

Ruellia menthoides (Nees) Hiern foi enquadrada no padrão de floração "anual intermediário" (Newstrom et al. 1994). Este padrão de floração assemelha-se ao padrão "cornucópia” de Gentry $(1974,1990)$, no qual a floração estende-se por alguns meses e pequenas abelhas estão entre os grupos de polinizadores, tal como as observadas em flores de $R$. menthoides por Lima (2001). Nos demais meses do ano, essa espécie produz flores cleistógamas (Lima et al. 2005) e, por isso, a sua frutificação também é contínua.

Os picos de floração observados no presente estudo foram verificados em períodos de transição entre estações, como observado em outras espécies por Morellato \& Leitão Filho (1996), na Mata Santa Genebra, em Campinas, SP. Ruellia brevifolia apresentou pico de floração na transição da estação úmida para seca, enquanto que $R$. subsessilis e $R$. menthoides apresentaram os picos de floração na transição da estação seca para úmida. Nesse último

Tabela 2. Número de flores abertas por planta por dia e porcentagem de plantas com apenas uma flor aberta por dia, em Ruellia brevifolia (Pohl) C. Ezcurra, Ruellia menthoides (Nees) Hiern e Ruellia subsessilis (Nees) Lindau.

Table 2. Number of open flowers per plant per day and percentage of plants with only one flower open per day in Ruellia brevifolia (Pohl) C. Ezcurra, Ruellia menthoides (Nees) Hiern and Ruellia subsessilis (Nees) Lindau.

Espécie (no de indivíduos)

Flores abertas por planta dia $^{-1}$ $\mathrm{n}$ o (amplitude)
Indivíduos com uma flor aberta por dia (\%)
01-15

$01-05$

01-06
60

84

81 
Tabela 3. Resultados do teste de germinação de sementes de Ruellia brevifolia (Pohl) C. Ezcurra, Ruellia menthoides (Nees) Hiern e Ruellia subsessilis (Nees) Lindau, oriundas de autopolinizações em flores casmógamas (AUT) e em flores cleistógamas (AUTcl).

Table 3. Results of the germination test of seeds of Ruellia brevifolia (Pohl) C. Ezcurra, Ruellia menthoides (Nees) Hiern and Ruellia subsessilis (Nees) Lindau, originated from self-pollination in chasmogamous flowers (AUT) and cleistogamous flowers (AUTcl).

\begin{tabular}{lcccc}
\hline Espécies & $\begin{array}{c}\text { Indivíduos } \\
\mathrm{n}^{\mathbf{0}}\end{array}$ & $\begin{array}{c}\text { Frutos } \\
\mathrm{n}^{\mathbf{0}}\end{array}$ & $\begin{array}{c}\text { Sementes } \\
\mathrm{n}^{\mathbf{0}}\end{array}$ & $\begin{array}{c}\text { Plântulas normais } \\
(\%)\end{array}$ \\
\hline R. brevifolia(AUT) & 4 & 5 & 52 & 67 \\
R. brevifolia(AUTcl) & 4 & 6 & 52 & 77 \\
R. menthoides (AUTcl) & 5 & 5 & 60 & 80 \\
R. subsessilis (AUT) & 6 & 6 & 77 & 83 \\
\hline
\end{tabular}

caso, as primeiras chuvas e o aumento da temperatura devem favorecer a floração dessas plantas (Morellato 1992, Morellato \& Leitão Filho 1996). Por outro lado, Croat (1975) verificou que a floração da maioria das espécies herbáceas, da Ilha de Barro Colorado, Panamá, é sazonal e mencionou que, no início da estação seca, a redução da umidade do solo e da atmosfera, bem como o aumento da luminosidade, parecem atuar como estímulos para a floração dessas plantas, assim como para a de $R$. brevifolia.

Os resultados sobre os períodos de floração obtidos no presente estudo acrescidos aos de Braz et al. (2000) e aos de Abreu \& Vieira (2004), também realizados na EPTEAMP, demonstram uma floração contínua e sobreposta, em diferentes graus, de cinco espécies ornitófilas de Acanthaceae, incluindo R. brevifolia e R. subsessilis. Essa floração resulta em um fornecimento de recurso floral (néctar), para os beija-flores, durante todo o ano e, por isso, as espécies dessa família parecem atuar, em conjunto, para a manutenção dessas aves na área de estudo, de maneira semelhante ao verificado por Stiles (1975), em estudos com espécies de Heliconia, e por Araújo et al. (1994), com espécies de Vriesea.

Sistema reprodutivo - A autocompatibilidade das espécies de Ruellia era esperada, especialmente devido à cleistogamia, e a maioria das espécies estudadas de sub-bosque tem apresentado autocompatibilidade (Kress \& Beach 1994). O comportamento reprodutivo de R. subsessilis assemelha-se ao de espécies "xenógamas facultativas", ou seja, ocorrência de polinização cruzada na presença de polinizadores e, na ausência deles, autopolinização (Kearns \& Inouye 1993). Em $R$. brevifolia, apesar da semelhança entre as frutificações nos testes de autogamia e alogamia, a autogamia prevalece, pois essa espécie produz flores CL, durante grande parte do ano e em sobreposição ao período de produção de flores CA (Lima et al. 2005). Esse comportamento reprodutivo assemelha-se ao de espécies "autógamas facultativas", ou seja, ocorrência de polinização cruzada em espécies que normalmente se autopolinizam (Kearns \& Inouye 1993). Piovano et al. (1995), após analisarem a razão pólen/óvulo em $R$. brevifolia, também sugeriram a "autogamia facultativa". Ruellia menthoides também comporta-se como "autógama facultativa", pois, além da autogamia em flores CA, produz flores CL praticamente o ano inteiro (Lima et al. 2005).

Nas três espécies de Ruellia, a autogamia parece ser vantajosa, considerando o histórico de desflorestamento da área de estudo. Durante esse processo pode ter ocorrido a extinção ou quase de muitos animais antófilos, como, por exemplo, os polinizadores de $R$. menthoides, raramente observados (Lima 2001). Em hábitats extremos ou marginais, a autogamia, incluindo a cleistogamia, tem sido considerada vantajosa devido à perda de polinizadores e às condições ambientais adversas, dentre outros fatores (Endress 1994, Briggs \& Walters 1997). Outra vantagem pode estar relacionada ao comportamento de visita dos beijaflores em flores de $R$. brevifolia e $R$. subsessilis. Nessas espécies, foram observadas, comumente, visitas ilegítimas, ou seja, essas aves perfuravam a base do tubo da corola para pilhar o néctar e, deste modo, não contatavam as estruturas reprodutivas (Braz et al. 2000, Lima 2001).

Dentre os mecanismos que dificultam a autopolinização espontânea, nas espécies estudadas, foram registrados a hercogamia, presente em grande parte das flores (Lima et al.2005), e a dicogamia parcial 
(protandria), verificada em $R$. brevifolia por Piovano et al. (1995) e por Lima et al. (2005). Além disso, a autopolinização espontânea deve ocorrer quando, durante a queda da corola e do androceu, há contato das anteras com o estigma, processo já observado em outras espécies de Ruellia (Long 1966, 1977). Esse processo tende a ser dificultado com o ensacamento das flores, pois a corola e o androceu ficam retidos junto ao restante da flor, e pode explicar, em parte, as frutificações mais baixas obtidas nas autopolinizações espontâneas, comparadas às autopolinizações manuais.

Segundo Ezcurra (1993), a autogamia sendo o principal mecanismo de reprodução, como parece ser para as espécies de Ruellia, tende a produzir populações locais morfologicamente distintas e erroneamente identificadas como espécies diferentes. Esta autora comentou que a dificuldade na taxonomia de muitas espécies de Ruellia pode estar relacionada às características do seu sistema reprodutivo. Ruellia menthoides, por exemplo, é morfologicamente semelhante à Ruellia kleinii C. Ezcurra \& Wassh. (Braz et al. 2002). Estudos sobre o sistema reprodutivo dessas espécies, incluindo polinizações interespecíficas, são importantes para esclarecer a relação entre elas.

Os resultados do presente estudo demonstraram a ausência de formação de sementes agamospérmicas nas espécies estudadas, contrariando as suposições de Braz et al. (2000). Essas autoras obtiveram altas porcentagens de frutos resultantes de autopolinização espontânea em $R$. brevifolia (85\%) e em $R$. subsessilis $(85 \%)$, levando-as a interpretá-las como resultantes de agamospermia.

As altas porcentagens de plântulas normais parecem indicar que as espécies não estão sob o efeito de depressão endogâmica (Richards 1997), o que era esperado devido à relevância da autogamia no sistema reprodutivo dessas plantas. Sementes viáveis, originadas de autopolinizações, já foram verificadas em Ruellia (Long 1964, 1971). Entretanto, estudos complementares sobre o vigor e estabelecimento de progênies devem ser realizados.

O modo de propagação vegetativa das espécies estudadas de Ruellia assemelha-se ao de várias outras espécies desse gênero (Lorenzi \& Souza 2001) e é comumente registrado em espécies de florestas úmidas (Murcia 1996). Os mecanismos assexuados, entretanto, têm sido negligenciados em estudos sobre sistemas reprodutivos. São necessários trabalhos enfocando a contribuição da reprodução clonal na manutenção das populações das espécies estudadas. Esses trabalhos deveriam incluir dados moleculares, com o objetivo de esclarecer a variabilidade genética das espécies, visando avaliar a efetividade dos polinizadores no fluxo gênico e o estado de conservação e fornecer subsídios adicionais para o manejo dessas plantas.

As espécies estudadas apresentaram diferentes estratégias reprodutivas, sexuadas - períodos distintos de picos de floração, flores CA autógamas ou alógamas, ornitófilas ou melitófilas e flores CL - e assexuada. Os resultados das estratégias sexuadas, entretanto, foram comuns a todas elas, ou seja, produção de frutos durante todo o ano. $\mathrm{O}$ balanço entre mecanismos variados de reprodução, como verificado nas espécies estudadas, parece favorecer a manutenção das populações em ambientes alterados, como é o caso das áreas de suas ocorrências na EPTEAMP.

Agradecimentos - À Capes, pela bolsa concedida à primeira autora, e ao $\mathrm{CNPq}$, pela bolsa concedida à segunda autora.

\section{Referências bibliográficas}

ABREU, C.R.M. \& VIEIRA, M.F. 2004. Os beija-flores e seus recursos florais em um fragmento florestal de Viçosa, sudeste brasileiro. Lundiana 5:129-134.

ARAÚJO, A.C., FISCHER, E.A. \& SAZIMA, M. 1994. Floração seqüencial e polinização de três espécies de Vriesea (Bromeliaceae) na região de Juréia, sudeste do Brasil. Revista Brasileira de Botânica 17:113-118.

BRAZ, D.M., VIEIRA, M.F. \& CARVALHO-OKANO, R.M. 2000. Aspectos reprodutivos de espécies de Acanthaceae Juss. de um fragmento florestal do Município de Viçosa, Minas Gerais. Revista Ceres 47:229-239.

BRAZ, D.M., CARVALHO-OKANO, R.M. \& KAMEYAMA, C. 2002. Acanthaceae da Reserva Florestal Mata do Paraíso, Viçosa, Minas Gerais. Revista Brasileira de Botânica 25:495-504.

BRIGGS, D. \& WALTERS, S.M. 1997. Plant variation and evolution. Cambridge University Press. Cambridge.

CASTRO, P.S. 1980. Influência da cobertura florestal na qualidade da água em duas bacias hidrográficas na região de Viçosa, MG. Dissertação de mestrado, Universidade de São Paulo, Piracicaba.

CROAT, T.B. 1975. Phenological behavior of habit and habitat classes on Barro Colorado Island (Panama Canal Zone). Biotropica 7:270-277.

DAFNI, A. 1992. Pollination ecology - a practical approach. Oxford University Press, Oxford.

ENDRESS, P.K. 1994. Diversity and evolutionary biology of tropical flowers. Cambridge University Press. Cambridge.

EZCURRA, C. 1993. Systematics of Ruellia (Acanthaceae) in southern South America. Annals of the Missouri Botanical Garden 80:784-845. 
GENTRY, A.H. 1974. Flowering phenology and diversity in tropical Bignoniaceae. Biotropica 6:64-68.

GENTRY, A.H. 1990. Evolutionary patterns in Neotropical Bignoniaceae. In Reproductive biology and evolution of tropical wood angiosperms (G. Gottsberger \& G.T. Prance, eds.). Memoirs of the New York Botanical Garden 55:118-129.

KEARNS, C.A. \& INOUYE, D.W. 1993. Techniques for pollination biologists. University Press of Colorado, Niwot.

KRESS, W.J. \& BEACH, J.H. 1994. Flowering plant reproductive systems. In La Selva - Ecology and natural history of a neotropical rain forest (L.A. MacDdade, K.S. Bawa, H.A. Hespenheide \& G.S. Hartshorn, eds.). University of Chicago Press, Chicago, p.161-182.

LEAL FILHO, N. 1992. Caracterização do banco de sementes de três estádios de uma sucessão vegetal na Zona da Mata de Minas Gerais. Dissertação de mestrado, Universidade Federal de Viçosa, Viçosa.

LIMA, N.A.S. 2001. Biologia reprodutiva de espécies de Ruellia (Acanthaceae) na Reserva Florestal Mata do Paraíso, Viçosa, MG. Dissertação de mestrado, Universidade Federal de Viçosa, Viçosa.

LIMA, N.A.S., VIEIRA, M.F., CARVALHO-OKANO, R.M. \& AZEVEDO, A.A. 2005. Cleistogamia em Ruellia menthoides (Nees) Hiern e R. brevifolia (Pohl) C. Ezcurra (Acanthaceae) em fragmento florestal do sudeste brasileiro. Acta Botanica Brasilica 19:443-449.

LONG, R.W. 1964. Biosystematic investigations in South Florida populations of Ruellia (Acanthaceae). American Journal of Botany 51:842-852.

LONG, R.W. 1966. Artificial interspecific hybridization in Ruellia (Acanthaceae). American Journal of Botany 53:917-927.

LONG, R.W. 1971. Floral polymorphy and amphimitic breeding systems in Ruellia caroliniensis (Acanthaceae). American Journal of Botany 58:525-531.

LONG, R.W. 1977. Artificial induction of obligate cleistogamy in species-hibrids in Ruellia (Acanthaceae). Bulletin of the Torrey Botanical Club 104:53-56.

LORENZI, H. \& SOUZA, H.M. 2001. Plantas ornamentais no Brasil: arbustivas, herbáceas e trepadeiras. Instituto Plantarum, Nova Odessa.

MACHADO, I.C.S. \& SAZIMA, M. 1995. Biologia da polinização e pilhagem por beija-flores em Ruellia asperula Lindau (Acanthaceae) na caatinga, nordeste brasileiro. Revista Brasileira de Botânica 18:24-33.

MAcDADE, L. 1985. Breeding systems of Central American Aphelandra (Acanthaceae). American Journal of Botany 72:1515-1521.

MORELLATO, L.P.C. 1992. Sazonalidade e dinâmica dos ecossistemas florestais da Serra do Japi. In História natural da Serra do Japi: ecologia e preservação de uma área florestal no sudeste do Brasil (L.P.C. Morellato, ed.). Editora da Unicamp, Campinas, p.98-110.
MORELLATO, L.P.C. \& LEITÃO FILHO, H.F. 1996. Reproductive phenology of climbers in a southeastern Brazilian forest. Biotropica 28:180-191.

MURCIA, C. 1996. Forest fragmentation and the pollination of neotropical plants. In Forest patches in tropical landscapes (J. Schellos \& R. Greenberg, eds.). Island Press, Washington, p.19-36.

NEES, C.G. 1847. Acanthaceae. In Flora brasiliensis (C.F.P. Martius, ed.). F. Fleischer, Lipsiae, v.9, p.1-164.

NEWSTROM, L.E., FRANKIE, G.W. \& BAKER, H.G. 1994. A new classification for plant phenology based on flowering patterns in lowland tropical rain forest trees at La Selva, Costa Rica. Biotropica 26:141-159.

PIOVANO, M.A., GALETO, L. \& BERNARDELLO, L.M. 1995. Floral morphology, nectar features and breeding system in Ruellia brevifolia (Acanthaceae). Revista Brasileira de Biologia 55:409-418.

RICHARDS, A.J. 1997. Plant breeding systems. $2^{\text {nd }}$ ed. Chapman \& Hall, London.

RIZZINI, C.T. 1992. Tratado de fitogeografia do Brasil: aspectos ecológicos, sociológicos e florísticos. Âmbito Cultural, São Paulo.

SIGRIST, M.R. \& SAZIMA, M. 2002. Ruellia brevifolia (Pohl) Ezcurra (Acanthaceae): fenologia da floração, biologia da polinização e reprodução. Revista Brasileira de Botânica 25:35-42.

SOARES JÚNIOR, F.J. 2000. Composição florística e estrutura de um fragmento de floresta estacional semidecidual na Fazenda Tico-Tico, Viçosa, MG. Dissertação de mestrado, Universidade Federal de Viçosa, Viçosa.

STACE, C.A. 1989. Plant taxonomy and biosystematics. $2^{\text {nd }}$ ed. Edward Arnold, London.

STILES, F.G. 1975. Ecology, flowering phenology, and hummingbird pollination of some Costa Rican Heliconia species. Ecology 56:285-301.

VELOSO, H.P., RANGEL FILHO,A.L.R. \& LIMA, J.C.A. 1991. Classificação da vegetação brasileira, adaptada a um sistema universal. Instituto Brasileiro de Geografia e Estatistica, Rio de Janeiro.

VIEIRA, M.F. \& CARVALHO-OKANO, R.M. 1996. Pollination biology of Mabea fistulifera (Euphorbiaceae) in southeastern Brazil. Biotropica 28:61-68.

VIEIRA, M.F. \& GRABALOS, R. 2003. Sistema reprodutivo de Oxypetalum mexiae Malme (Asclepiadaceae), espécie endêmica de Viçosa, MG, Brasil, em perigo de extinção. Acta Botanica Brasilica 17:137-145.

VIEIRA, M.F. \& LIMA, N.A.S. 1997. Pollination of Echinodorus grandiflorus (Alismataceae). Aquatic Botany 58:89-98.

VIEIRA, M.F. \& SHEPHERD, G.J. 2002. Oxypetalum banksii subsp. banksii: a taxon of Asclepiadaceae with an extragynoecial compitum. Plant Systematics and Evolution 233:199-206. 